\title{
The design of a circulating water pump for a power plant based on CFD
}

\author{
Wanbo Chen ${ }^{1, *}$ \\ ${ }^{1}$ Jiangxi Ganneng Co.,Ltd., 330096 No. 199 torch street, Nanchang City, China
}

\begin{abstract}
Because of the head and efficiency of the circulating water pump in a power plant could not satisfy application requirement, the guide vane and impeller were optimized according to the CFD method. The optimization of the mixed-flow pump effectively improves the flow state at the design and high discharge conditions. Finally, the CFD results show that the head is improved by $3.49 \mathrm{~m}$ and the efficiency is improved by $3.16 \%$ after the optimization, which has met the use requirements.
\end{abstract}

\section{Introduction}

Mixed-flow pump is a kind of structure and performance between the axial pump and centrifugal pump type, its high efficiency, wide range of operation, widely used in agriculture, municipal, power plants and other pumping station projects, in recent years in nuclear power, ship water injection propulsion has also been widely used.

As the core component of mixed-flow pump, the performance of the impeller and guide vane is very important for the efficient and stable operation of the mixed-flow pump. At present, most of the optimization of mixed-flow pumps is still through CFD numerical simulation methods, FelixA.Mugglietal using the large vortex simulation method, the characteristics of the abnormal flow inside a mixed-flow pump are analyzed, and the unstable flow structure inside the pump under partial operating conditions is analyzed in depth. B.P.M. Vanesch carried out a non-normal, non-stick calculation of industrial mixed-flow pumps, and the results showed that the non-steady-state calculation of impellers and worm shells could better reflect the overall performance of the pump than just one of them alone. L. Yabin investigated the energy performance and tip leakage vortex in a mixed-flow pump as turbine (PAT) at pump mode by numerical method validated by experiment measurement. Kim used computational fluid dynamics (CFD) and design of experiment (DOE) to study the shape of a mixed-flow pump impeller and its flow characteristics as a function of the specific speed.

In this paper, a circulating water pump was used as a research object to establish a full-fluid domain model of real machine size through CFX numerical simulation technology. The mixed-flow pump was geometrically modified by UG. The study has improved the hydraulic efficiency, at the same time for mixed-flow pump optimization to provide reference.

\section{Physical model}

\subsection{Geometric model}

In order to reduce geometric loss and accurately simulate the shape of the real pump, UG software is used to model the mixed-flow pump impeller blade, guide vane and other overflow components. The 3D model of the pump calculation domains is shown in Fig.1.Different parts of the modified comparison shown in Fig.2.Several key parameters of mixed-flow pump are listed in Table 1.

Table 1. Calculate the parameters

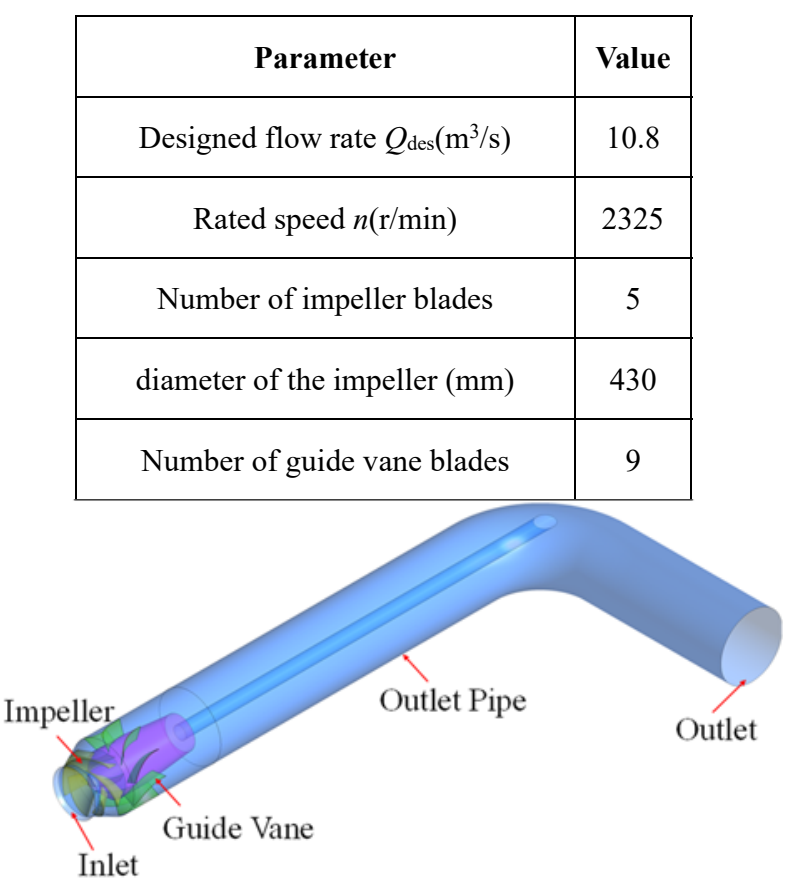

Fig. 1. 3D diagram of Mixed-flow Pump 


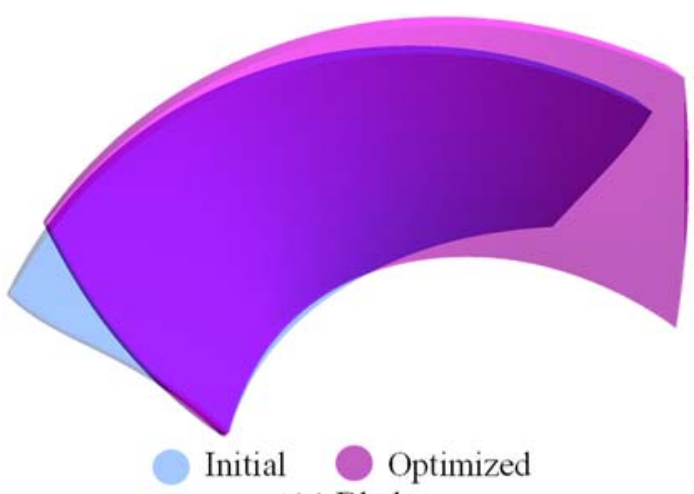

(a) Blade

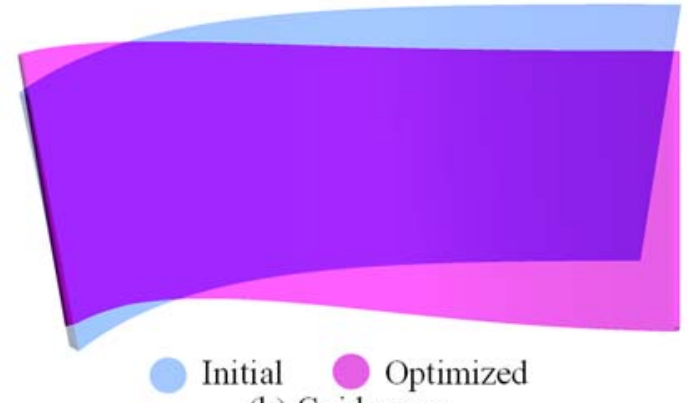

(b) Guide vane

Fig 2.Comparison of modification in different parts

\subsection{Meshgeneration}

In order to accurately simulate the internal flow of each flow component of the pump, and to minimize errors in numerical calculations, the meshing of each overflow component is carried out by using a high-precision mesh. The refined mesh in the boundary layer and tip region of the impeller section is shown in Fig.3. The total number of grid nodes is 3.5 million. The automatic near-wall treatment was employed to suit the turbulence model and the $\mathrm{y}+$ of the impeller section on the blade surface is below 100.Control equations and boundary conditions are given in Table.2.

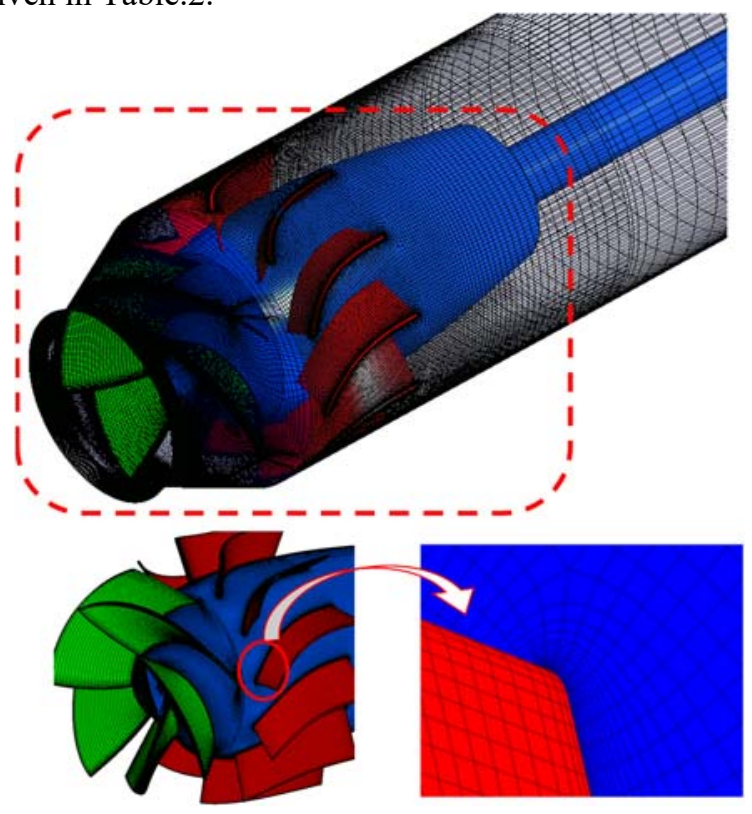

Fig 3. Whole computational domain and domain discretization

Table2. Boundary conditions

\begin{tabular}{|c|c|}
\hline \multirow{2}{*}{ CFD solver } & ANSYS CFX \\
\hline \multirow{2}{*}{ Inlet } & total pressure \\
\cline { 2 - 2 } Outlet & $5 \%$ turbulence intensity \\
\hline Boundary details & Flow \\
\hline Divergent method & second-order no-slip. \\
\hline Turbulence & Shear Stress Transport (SST) \\
\hline Convergence control & $\leq 10^{-4}$ \\
\hline
\end{tabular}

\section{Result and discussion}

Fig 4. shows the energy performance curve with the mixed-flow pump. Those curves are smoothed by Bessel method. It is shown in these figure that the effect of the retrofit of the mixed-flow pump. When the flow condition changes from $0.88 \mathrm{Q}_{\mathrm{des}}$ to $1.16 \mathrm{Q}_{\mathrm{des}}$, the pump head and efficiency drops with the retrofit.

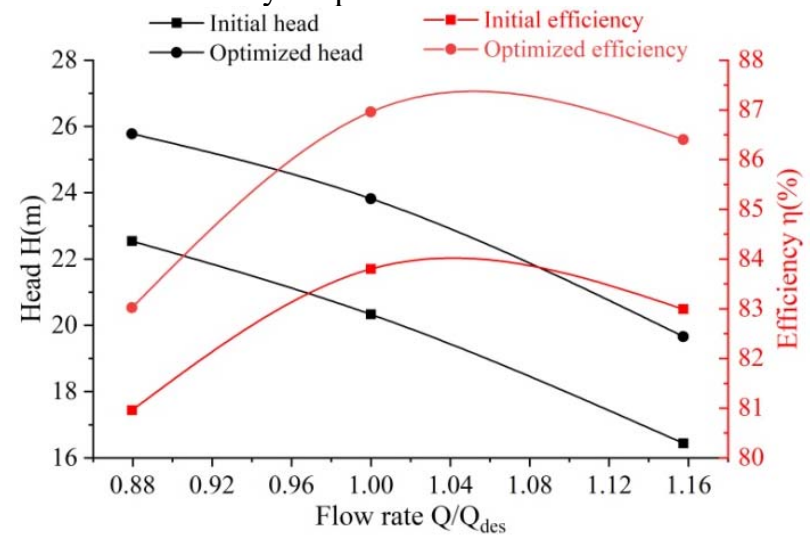

Fig 4. Comparison of head and efficiency

In order to compare the internal flow characteristics of the mixed-flow pumps, the distribution of turbulent kinetic energy and velocity flow at 30\%, 50\% and 70\% of the cylindrical unfold surface in the impeller and guide vane were shown in Fig 5. Turbulent kinetic energy is an indicator of the development or decline of turbulence in time and space, and also a sign of flow stability. The comparison found that the distribution of turbulent kinetic energy under the small flow conditions after optimization did not change much, the turbulent kinetic energy in the guide vane flow channel decreased, the high-intensity turbulence concentration area existed on the back of the guide vane under the pre-optimization design flow and high flow, and the distribution range of high turbulent kinetic energy in the optimized guide vane flow channel was significantly reduced. 
Turbulence Kinetic Energy $\left(\mathrm{m}^{2} \mathrm{~s}^{-2}\right)$

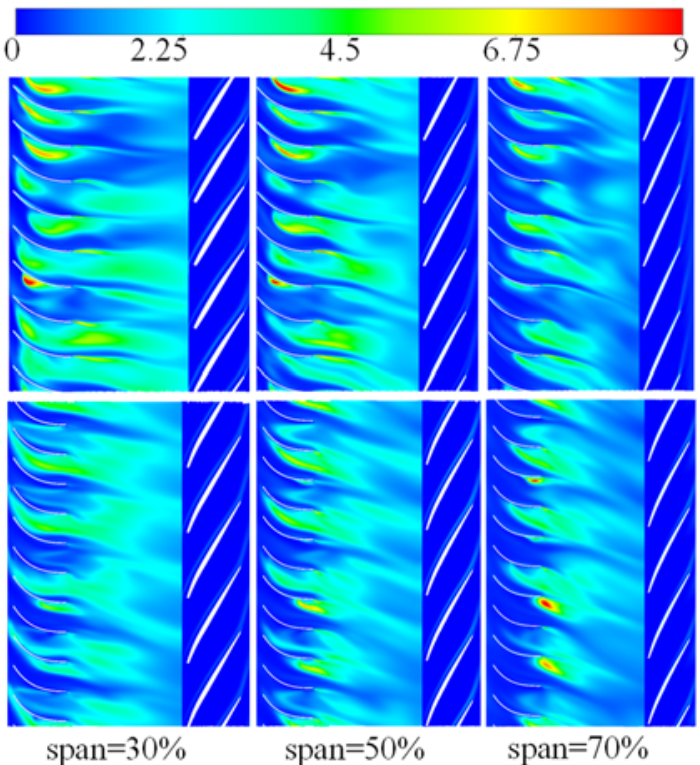

span $=30 \%$

span $=50 \%$

(a) $0.88 \mathrm{Q}_{\mathrm{des}}$

Turbulence Kinetic Energy $\left(\mathrm{m}^{2} \mathrm{~s}^{-2}\right)$

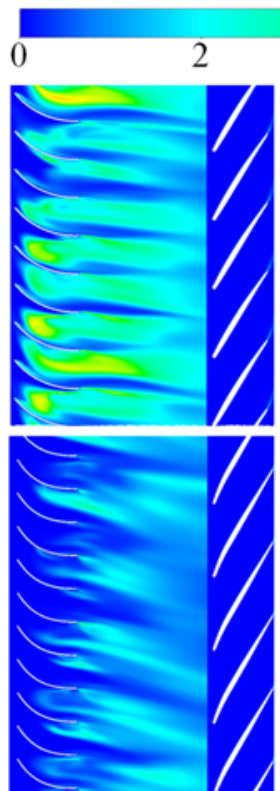

span $=30 \%$
4
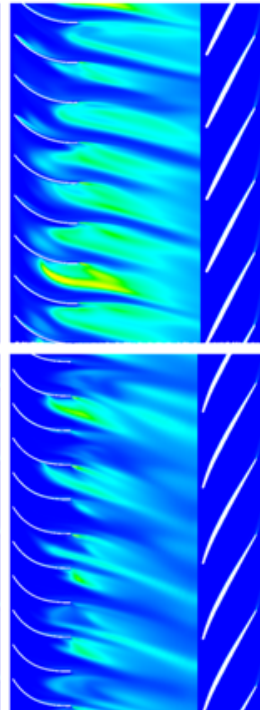

span $=50 \%$

(b) $1.0 Q_{\text {des }}$
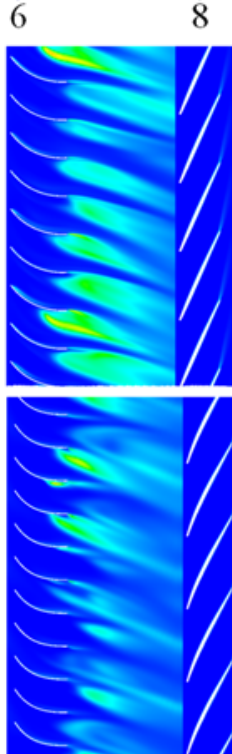

$\operatorname{span}=70 \%$
Turbulence Kinetic Energy $\left(\mathrm{m}^{2} \mathrm{~s}^{-2}\right)$

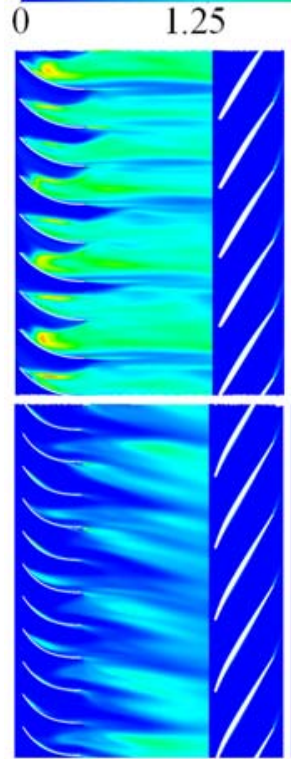

span $=30 \%$
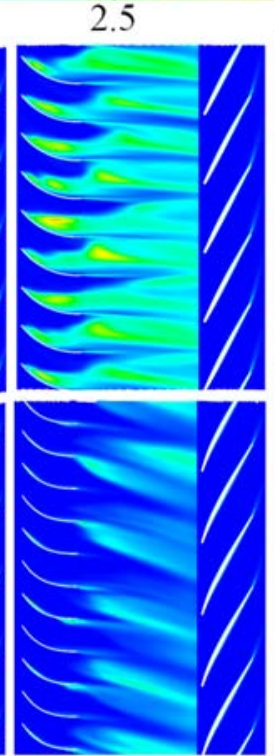

span $=50 \%$

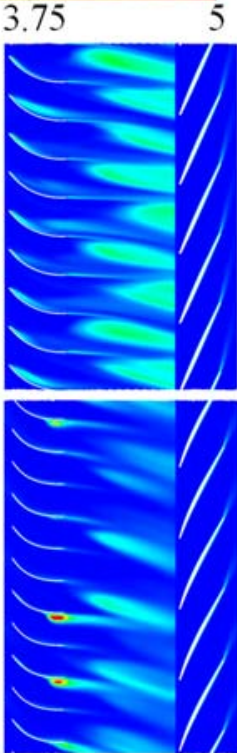

$\operatorname{span}=70 \%$

\section{(c) $1.16 \mathrm{Q}_{\mathrm{des}}$}

Fig 5. Comparison of the turbulence kinetic energy before and after optimization

As shown in the Fig 6, the surface pressure distribution of the impeller. As can be seen from the figure, the lowest pressure value appears near the blade suction surface area, and the highest pressure value appears near the blade pressure surface area. After the optimization of the mixed flow pump, the pressure distribution gradient of the blade surface is small, the area is relatively homogeneity, and the pressure difference of the work surface is small, which indicates that the flow rate is more homogeneity and the effect is better.

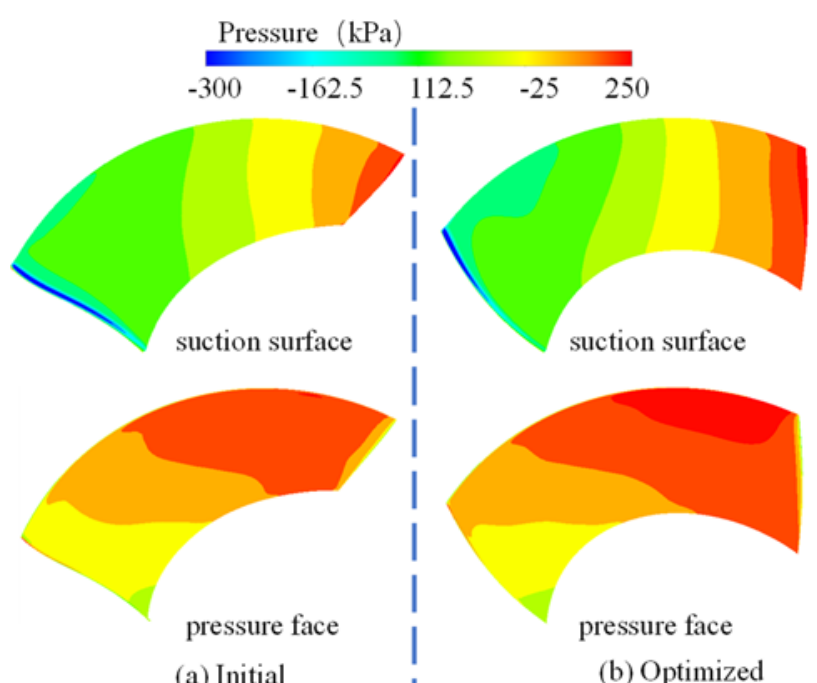

(a) Initial (b) Optimized

(1) $\mathrm{Q}=0.88 \mathrm{Q}_{\mathrm{des}}$ 


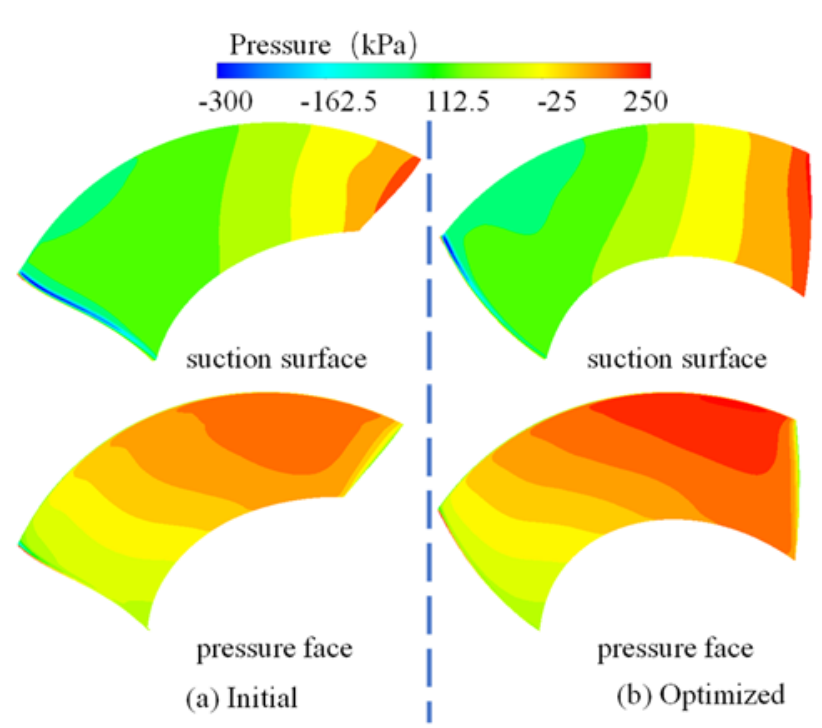

(2) $\mathrm{Q}=1.0 \mathrm{Q}_{\text {des }}$

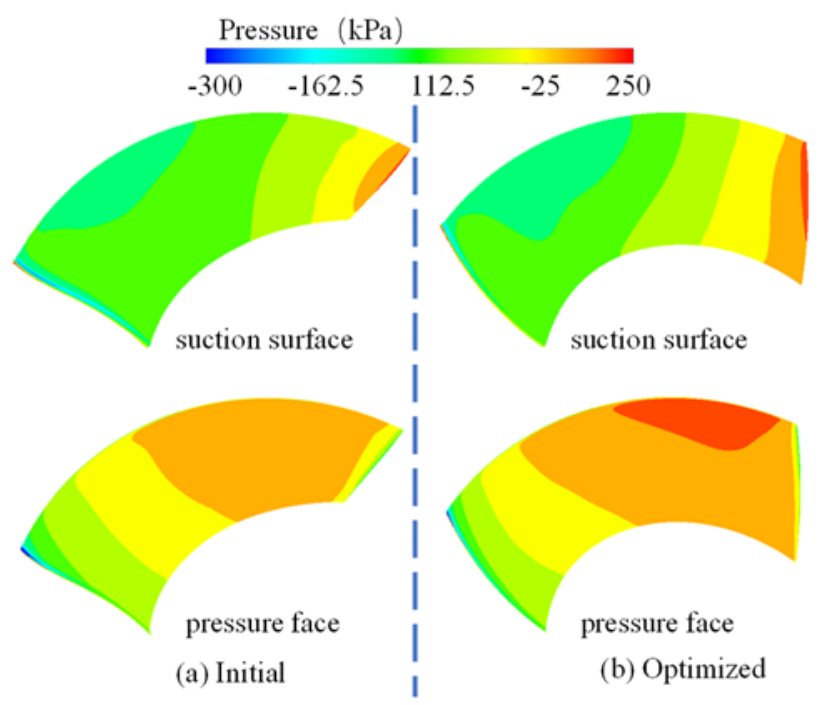

(3) $Q=1.16 Q_{\text {des }}$

Fig 6.Pressure distribution on blade surface

Fig 7. is the streamline of the impeller and guide vane at $1.0 \mathrm{Q}_{\text {des. }}$. As can be seen from the figure, after optimizing the mixed-flow pump, the streamline distribution after the guide vane near the hub becomes homogeneity, reducing the occurrence of undesirable states such as turbulence.

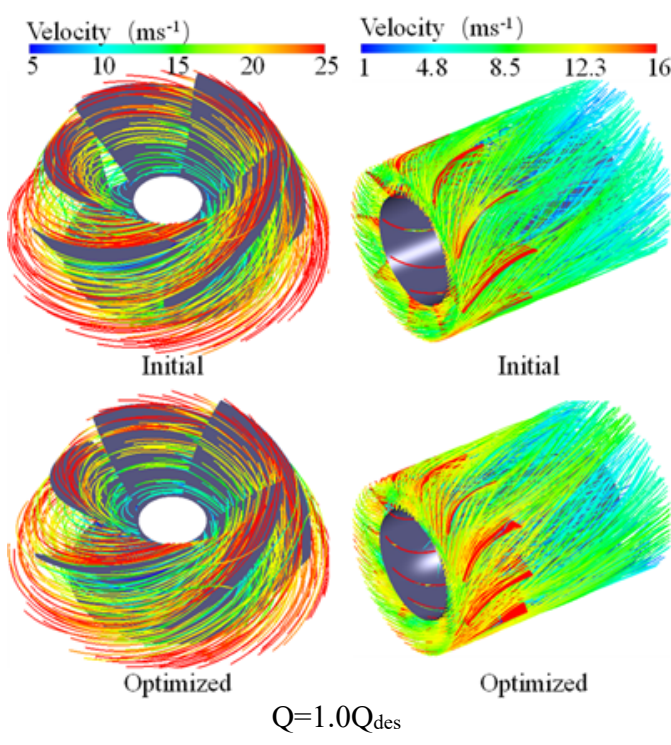

Fig 7. 3D streamlines in impeller and guide vane

In summary, the flow patterns of the pump are improved by optimizing the mixed-flow pump, and the vortex of the guide position is reduced, so that the mixed-flow pump has good hydraulic performance.

\section{Conclusions}

In this study, design optimization of a circulating water pump impeller and guide vane is performed to improve its efficiency and suction performance.

(1) After the optimization of the mixed-flow pump, the results show that the head is improved by $3.49 \mathrm{~m}$ and the efficiency is improved by $3.16 \%$.

(2)Comparing the distribution of turbulent kinetic energy within each flow rate impeller and the guide vane flow channel, the optimized mixed-flow pump can effectively reduce the energy loss between the design flow rate and the high flow rate.

(3) Comparing the surface streamline of the impeller at $1.0 \mathrm{Q}_{\text {des. }}$. The distribution of streamline turns more homogeneity.

\section{References}

1. N. Dash, A. Kumar Roy, K. Kumar. Materials today: proceedings 5, 8 (2018)

2. F. A. Muggli,P. Holbein,P. Dupont. Journal of Fluids Engineering, 124,10 (2002)

3. v. B. P.M. Esch, N. P. Kruyt. Journal of Fluids Engineering, 123, 9(2001)

4. L. Yabin, H. Yadong, T. Lei. Energy,(2020) 